\title{
Biosimilars and patient safety risk: promoting policy protections in the health delivery system
}

Bryan A. Liang ${ }^{1,2,3}$, Timothy Mackey ${ }^{1,4}$

Institute of Health Law Studies, California Western School of Law, San Diego, CA, USA

Department of Anesthesiology, University of California, San Diego School of Medicine, San Diego, CA, USA

San Diego Center for Patient Safety, University of California, San Diego School of Medicine, San Diego, CA, USA

Joint Program in Global Health, University of California San Diego-San Diego State University, San Diego, CA, USA

\section{Introduction}

Biologic drugs are large, complex, biotechnology molecules made from living cell lines. These formulations represent the future of pharmaceutical treatment. Indeed, it is estimated that these drugs will account for more than half of newly approved drugs worldwide [1], and by 2015, 8 of the 10 top-selling medications are predicted to be biologics [2] with global spending reaching some $\$ 200$ billion [3].

However, biologics are expensive. In developed countries, costs can rise as high as $\$ 10,000$ per month [4]. Yet price reductions and increased access can occur with generic drug entry. This pricing dynamic may occur with the entry of biosimilars, non-innovator forms of the drug that mimic and compete with the original biologic.

With global increases in health care costs and current economic challenges, there is tremendous interest in biosimilars. Estimates are that global biosimilars spending will increase $700 \%$ to $800 \%$ in the 2010 to 2015 time frame [3], replacing a significant fraction of the approximately $\$ 80$ billion in global sales of branded biologic drugs expected to be coming off patent by 2015 . Hence, biosimilars are seen to have tremendous potential to improve quality and quantity of life.

However, there is a significant risk with these products: unwanted immunogenicity. Ensuring provider information and vigilance to immunogenicity and its related adverse reactions is an important patient safety concern. Public health and policy goals of reduced costs and improved access can bring the potential benefits of biologics to greater patient populations only if biosimilars are safely integrated into delivery systems. 


\section{Similar, not identical}

The inherent complexity of biologics and their manufacture can create potential differences across biosimilar versions of the original product. Because of the differences in production as well as the unique cellular source of these drugs, it is nearly impossible to make truly identical copies of a protein using two different production cell lines [5]. This diversity is present to an even greater degree across cell lines from different living organisms, such as bacteria, yeast, mammalian organs, and other sources. Further, cell lines do not always create the full, functional protein efficiently. Indeed, the biologic "product" from a cell line is actually a heterogeneous and complex mix of materials that includes the desired product as well as side products and cell materials [6].

Consequently, even if the DNA sequence coding for a protein is identical between original biologics and biosimilar forms, a different product may result from use of different cell lines. Accordingly, complete characterization of the biologic products from a specific cell line creating a particular biologic is often not possible. The complexity of the biologic molecule, its sensitivity to production, and the challenges associated with characterization result instead in its being defined in terms of its manufacturing method [7].

This situation should be contrasted with small molecule, chemical drugs. For these products, generic producers can make identical copies either by using the same known chemical processes or through independently developed processes [7]. Indeed, atom-by-atom comparisons between the original product and the generic version are possible through a wide array of chemical and functional tests, to verify the products have identical chemical compositions. Purification processes can be performed on discrete, individual molecules, resulting in a highly uniform composition.

In comparison, a biosimilar by its very nature - large size, complexity, and living organism production - will be different from the original form. This makes both originator and biosimilar biologic drug production much more challenging than small molecule, generic drug production [8].

\section{Immunogenicity}

Due to the large size of biologics, there is one central concern that is not present for chemical medicines: immunogenicity, i.e., the potential for the product to induce an adverse immunologic reaction [9]. Reactions can be severe and represent a major medical emergency, yet are notoriously difficult to predict.

For example, a EU-licensed version of erythropoietin caused severe immunogenicity reactions while no USA cases were observed $[1,10]$. Through cooperative licensing, both Amgen and Johnson \& Johnson purportedly used the same method to produce Amgen's original erythropoietin drug. The former was produced in the USA and known as Epogen ${ }^{\circ}$, while the latter was produced in the EU and known as Eprex ${ }^{\oplus}[1]$.

Johnson \& Johnson made several changes to the formulation of Eprex ${ }^{\bullet}$ that were initially considered minor [1]. However, two years after Johnson \& Johnson made these changes, patients taking Eprex ${ }^{\otimes}$ in the EU began developing a rejection reaction to the drug that resulted in pure red cell aplasia, with patients experiencing severe, life-threatening cessation of red blood cell production. There were no reports of any increased incidence of pure red blood aplasia among patients taking Epogen ${ }^{\bullet}$ [1].

Investigations revealed Eprex ${ }^{\circ}$ had a different immunogenicity profile than Epogen ${ }^{\circ}$ [10]. Furthermore, the new observed immunogenicity also resulted in antibody creation against the patients' own naturally-occurring erythropoietin. It also resulted in cross-reactivity responses to other pharmaceutical forms of erythropoietin, including Epogen ${ }^{\circledR}$, resulting in an inability of patients to rely on other forms of the drug. The outcome was that patients could not use commercial versions of 
exogenous erythropoietin or their own native forms to produce new red blood cells. Several patients died, while others became permanently transfusion-dependent. After more than a decade of research, full explanations are still elusive, with a change in rubber stopper, different carbohydrate structures, route of administration, a change in the stabilizer as suggested factors in Eprex ${ }^{\circledast}$ immunogenicity [11].

\section{Patient safety}

Biosimilar immunogenicity represents significant patient safety concerns. Systemic approaches that employ public and private sector cooperation and partnerships for proactive warnings, education, and identification of immunogenicity are important to mitigate the potential for patient harm.

Hence, addressing the patient safety concerns around biosimilars should be the priority when considering integration of these molecules into the medical arsenal. The USA Institute for Safe Medication Practices (ISMP) "High-Alert Medications" approach can serve as a foundation for integrating biosimilars into a health delivery system [12]. Local public health departments should leverage established provider communications systems to disseminate information about biosimilars as highalert medications, accompanied by publicly available ISMP tools and recommendations, including locked cabinets, sign-out forms, and other methods to alert providers and highlight risks of these drug forms.

Providers and public health departments should coordinate with biosimilar manufacturers to ensure biosimilar packaging and labeling facilitate proactive safety and risk messaging. Further, global manufacturing and distribution firms with experience in immunogenicity can provide technical information to contribute to provider education and communication, and working with public health departments, can create culturally-competent materials to reach patients and the community regarding the safety issues surrounding biosimilar forms.

Cooperative, public-private rapid reporting and immunogenicity identification systems should also be created. Here, global pharmaceutical firms have extensive experience in adverse event reporting systems across health care delivery systems. This experience in the context of public health-industry communications infrastructure and surveillance can provide an opportunity to cooperatively design effective systems of communication. This infrastructure can also be extended beyond biosimilar immunogenicity warnings and can incorporate additional public health concerns, including drug recalls, counterfeit drug warnings, food safety warnings, post-market surveillance, and disaster communications.

Similarly, joint public-private responses to an immunogenicity reporting can occur. Dissemination of information to the community using proactively designed channels employing culturally-competent messaging and methods can be efficient and rapid, an important consideration for immunogenicity events. In addition, such a system may leverage global firm knowledge in use of electronic auxiliary labels and automated alerts, if and when local health IT systems could accommodate it. In addition, direct-to-consumer communications of adverse events/risks, employing Internet, social networks, and mobile handset messaging and notification technology, can also be leveraged for patient safety purposes if done appropriately [13,14].

Standardization of biosimilar purchase and use is also an important patient safety intervention. Because current infrastructural differences between and within systems for naming biosimilars globally have not adopted unique names or designations, this makes identification of biosimilar source a tremendous challenge [1]. Governments and/or facilities using biosimilars should consider purchasing only one version to provide prompt identification and forensic analysis of any product that can result in immunogenicity [15]. 


\section{Conclusion}

Biosimilars have tremendous potential to increase access and reduce costs for cutting-edge biologic drugs. However, paramount to any integration of biosimilars into the health delivery system is safety: systemically addressing unwanted immunogenenicity. By integration of public and private sector efforts and resources, the benefits of biosimilars can expand while ensuring safety and an infrastructure that can grow and modernize with the global advancement of therapeutic targeted therapies in the biologics sphere.

\section{References}

1. Liang B. Regulating Follow-On Biologics. Harv J Legis 2007; 44: 363-471

2. Silverman E. Drug pipeline loses pressure. Manag Care 2010; 19: 23-6

3. Comer B. Biosimilars Spend to Reach $\$ 2.5$ Bln by 2015. blog.pharmexec.com, 2011. Available at: http://blog.pharmexec.com/2011/05/18/biosimilars-spend-to-reach-2-5-bln-by-2015-ims [last accessed Mar. 27, 2012]

4. Maiese BA, Lee EH, Toscani M. Follow-on biologics \& biosimilars literature review: the current landscape and implications of recent healthcare legislation for the U.S. market. SpecialtyPharmaJournal.com, 2011. Available at: http://www.specialtypharmajournal.com/index. php?option=com_content\&view=article\&id=2816:follow-on-biologics-a-biosimilars-literaturereview-the-current-landscape-and-implications-of-recent-healthcare-legislation-for-the-us-market- \&catid=425: catbiosimilars\&Itemid=845 [last accessed Mar. 8, 2012]

5. Roger SD. Biosimilars: How similar or dissimilar are they? Nephrology 2006; 11: 341-6

6. Statement of Jay P. Siegel, M.D., Johnson \& Johnson. Before the Senate Committee on Health, Education, Labor, and Pensions: Follow-on Biologics, 2007 Available at: http://www.help.senate. gov/old_site/Hearings/2007_03_08/Siegel.pdf [last accessed Mar. 12, 2012]

7. Leuenberger-Fisher MR. The road to follow on biologics: are we there yet? Biotech Law Rep 2004; 23: 389-401

8. EuropaBio. Guide to biological medicines: a focus on biosimilar medicines [Internet]. europabio.org. Available at: http://www.europabio.org/sites/default/files/report/guide_to_biological_ medicines_a_focus_on_biosimilar_medicines.pdf [last accessed Mar. 12, 2012]

9. Chamberlain P. Immunogenicity of therapeutic proteins. Part 1: Causes and clinical manifestations of immunogenicity. Reg Rev 2002; 5: 5-9

10. Bennett CL, Cournoyer D, Carson KR, et al. Long-term outcome of individuals with pure red cell aplasia and antierythropoietin antibodies in patients treated with recombinant epoetin: a followup report from the Research on Adverse Drug Events and Reports (RADAR) Project. Blood 2005; 106: 3343-7

11. Schellekens H, Jiskoot W. Eprex-associated pure red cell aplasia and leachates. Nat Biotechnol 2006; 24: 613-4

12. ISMP. ISMP's High-Alert Medications List. ismp.org Available at: http://www.ismp.org/Tools highAlertMedications.asp [last accessed Mar. 22, 2012]

13. Mackey TK, Liang BA. The global counterfeit drug trade: Patient safety and public health risks. $J$ Pharm Sci 2011; 100: 4571-9

14. Liang BA, Mackey T. Reforming direct-to-consumer advertising. Nat Biotechnol 2011; 29: 397-400

15. Liang BA, Mackey T. Emerging patient safety issues under health care reform: follow-on biologics and immunogenicity. Ther Clin Risk Manag 2011; 7: 489-93 